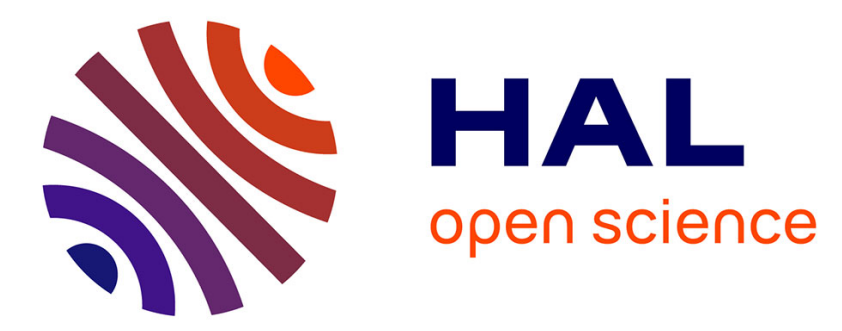

\title{
Probing activation energy barrier distribution for reversal of strongly exchange-coupled magnetic multilayer thin films
}

O Ozatay, Thomas Hauet, Florez S.H., J. A. Katine, A Moser, J.-U Thiele, L Folks, B. D. Terris

\section{To cite this version:}

O Ozatay, Thomas Hauet, Florez S.H., J. A. Katine, A Moser, et al.. Probing activation energy barrier distribution for reversal of strongly exchange-coupled magnetic multilayer thin films. Applied Physics Letters, 2009, 10.1063/1.3250924 . hal-01345318

\author{
HAL Id: hal-01345318 \\ https://hal.science/hal-01345318
}

Submitted on 13 Jul 2016

HAL is a multi-disciplinary open access archive for the deposit and dissemination of scientific research documents, whether they are published or not. The documents may come from teaching and research institutions in France or abroad, or from public or private research centers.
L'archive ouverte pluridisciplinaire HAL, est destinée au dépôt et à la diffusion de documents scientifiques de niveau recherche, publiés ou non, émanant des établissements d'enseignement et de recherche français ou étrangers, des laboratoires publics ou privés. 


\title{
Probing activation energy barrier distribution for reversal of strongly exchange-coupled magnetic multilayer thin films
}

\author{
O. Ozatay, ${ }^{1,2, a)}$ T. Hauet, $^{2}$ S. H. Florez, ${ }^{2}$ J. A. Katine, ${ }^{2}$ A. Moser, ${ }^{2}$ J.-U. Thiele, ${ }^{2}$ L. Folks, ${ }^{2}$ \\ and B. D. Terris ${ }^{2}$ \\ ${ }_{1}^{1}$ Department of Physics, Bogazici University, Bebek 34342, Istanbul, Turkey \\ ${ }^{2}$ Hitachi Global Storage Technologies-San Jose Research Center, San Jose, California 95135, USA
}

(Received 23 August 2009; accepted 26 September 2009; published online 27 October 2009)

\begin{abstract}
We demonstrate a measurement technique with zero-applied magnetic field to deduce and spatially map the activation energy barrier distribution of strongly exchange-coupled magnetic-multilayer thin films, which is otherwise inaccessible with conventional methods in the presence of an applied magnetic field. Our technique involves the analysis of magnetic force microscopy images of magnetic microwires, whose magnetizations have been subject to thermal decay due to Joule heating from applied nanosecond scale current pulses. Fitting the results of such measurements on $\mathrm{CoNi} / \mathrm{Pd}$ magnetic-multilayer microwires to a modified Arrhenius-Neel formalism yields an energy barrier distribution with $8 \%$ sigma, in good agreement with complementary fits of the switching-field-distribution measurements on patterned $\mathrm{CoNi} / \mathrm{Pd}$ magnetic-multilayer islands. (C) 2009 American Institute of Physics. [doi:10.1063/1.3250924]
\end{abstract}

In order to achieve reliable operation of nanoscale magnetic devices, it is paramount to understand the role of spatial inhomogeneity of intrinsic magnetic properties, such as magnetization, exchange, anisotropy, and activation energy for reversal in the overall performance of these devices. This evaluation is also necessary to separately diagnose the complementary contributions to the element-to-element performance variations from extrinsic (i.e., patterning induced) factors, such as ion-mill edge damage ${ }^{1}$ and sidewall oxidation effects. ${ }^{2}$

In the case of an array of magnetic dots, one of the most important consequences of a finite distribution of intrinsic magnetic properties is the dot-to-dot reversal field variation. This so-called switching field distribution (SFD) can lead to the misregistration of the information in bit-patterned media recording. Gathering information about the SFD of strongly exchange-coupled films is of primary importance, since such materials offering large exchange lengths $\left(l_{\mathrm{ex}}\right.$ $=\sqrt{A / 2 \pi M_{s}^{2}}=20-25 \mathrm{~nm}$ for $(\mathrm{CoNi} / \mathrm{Pd})$ multilayers with an effective exchange constant $A=3-6 \times 10^{-6} \mathrm{ergs} / \mathrm{cm}(3-6$ $\left.\times 10^{-11} \mathrm{~J} / \mathrm{m}\right)$ (Ref. 3) and measured $M_{s}=250 \mathrm{emu} / \mathrm{cm}^{3}$ $(0.31 \mathrm{~T})$ [see Fig. 1(d)] are the ones that will most likely adapt a stable single-domain magnetic configuration when patterned into nanoscale memory devices. While in weakly exchange-coupled continuous granular magnetic media, the squareness of the magnetization reversal hysteresis loops directly reflects the SFD, such information is not directly available from the hysteresis loop measurements of strongly exchange-coupled magnetic thin films. Indeed the reversal mechanism of strongly exchange-coupled film is that of nucleation in weakest anisotropy regions followed by rapid domain wall propagation. ${ }^{4}$ Therefore it is not possible to deduce the reversal energy barrier distribution of such regions from a direct macroscopic study of the magnetic properties. Thomson et $a l .{ }^{4}$ have recently shown that the SFD of an array of strongly exchange-coupled magnetic nanoislands

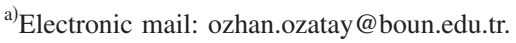

can be used to get a quantitative estimation of the intrinsic anisotropy distribution of the full film. This was achieved by fitting a modified Sharrock formalism ${ }^{5}$ to the switching field data for patterned nanoislands of $\mathrm{Co} / \mathrm{Pd}$ multilayers with different element sizes. However such a determination a priori necessitates the full electron beam (e-beam) patterning of the film to determine the intrinsic anisotropy distribution and energy barrier distribution.

Here we demonstrate an alternative technique, which requires only a modest photolithography patterning and in addition gives an actual spatial mapping of the intrinsic energy barrier distribution using magnetic force microscopy (MFM) imaging. This technique entails imaging of the gradual magnetization decay due to current pulse-induced Joule heating in patterned microwires. After applying a systematic threshold filtering to the acquired images to quantify the amount of

(a)
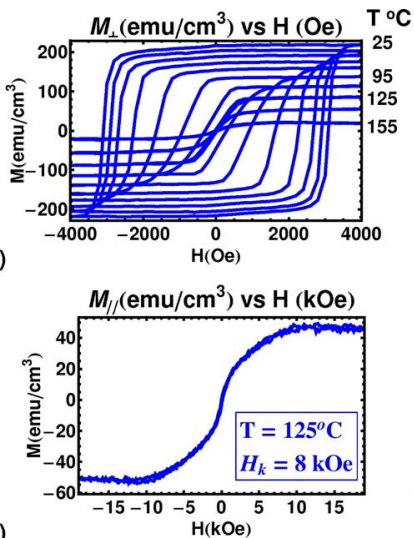

(c)

FIG. 1. (Color online) VSM measurements of the bulk properties of $\mathrm{CoNi} / \mathrm{Pd}$ multilayer thin film. (a) Magnetization (m) vs magnetic field (h) applied perpendicularly to the film plane hysteresis loops measured at temperatures from 25 to $150{ }^{\circ} \mathrm{C}$. (b) The coercive fields (zero crossing points) extracted from $\mathrm{m}$ vs $\mathrm{h}$ measurements shown in (a) as a function of temperature from 25 to $150{ }^{\circ} \mathrm{C}$. (c) In-plane hard axis loop measured at $125^{\circ} \mathrm{C}$. (d) Magnetization vs temperature measured in the presence of a $1 \mathrm{kOe}$ out-ofplane magnetic field (red dots) and in the absence of any applied magnetic field (blue dots). 
magnetization decay, the resulting data can then be fit to a modified Arrhenius-Neel exponential decay model (as described below) assuming that the energy barriers for reversal have a Gaussian distribution and each nucleation volume behaves as a Stoner-Wohlfarth particle. In this experiment we have applied this technique to determine the activation energy barrier distribution for reversal in $(\mathrm{CoNi} / \mathrm{Pd})$ perpendicular anisotropy magnetic multilayers.

The magnetic multilayers have been sputter deposited onto a $\mathrm{Si} / \mathrm{SiO}_{2}$ substrate with a structure of $\mathrm{Ta}(1.5)$ / $\operatorname{Pd}(3) /\left[\mathrm{Co}_{55} \mathrm{Ni}_{45}(0.22) / \mathrm{Pd}(1.2)\right] \times 22$ repeats $/ \mathrm{Pd}(2)$ where all thicknesses are in nanometers. Temperature dependence of perpendicular magnetic field hysteresis loops, coercive field, and saturation magnetization of the full film were measured with a vibrating sample magnetometer from room temperature up to the Curie temperature of $175^{\circ} \mathrm{C}$ as shown in Figs. 1(a), 1(b), and 1(d) respectively together with an inplane hysteresis loop measurement at $125^{\circ} \mathrm{C}$ in Fig. 1(c). It can be seen from Fig. 1(a) that at around $125^{\circ} \mathrm{C}$ the nucleation field for the full film drops down to zero together with the coercive field [as seen in Fig. 1(b)]. Figure 1(d) indeed shows that even though the Curie temperature is $175^{\circ} \mathrm{C}$ as measured with $1 \mathrm{kOe}\left(79.6 \times 10^{3} \mathrm{~A} / \mathrm{m}\right)$ magnetic field applied perpendicular to the film plane, the remanent magnetization goes through a gradual relaxation above $100{ }^{\circ} \mathrm{C}$ reaching the zero point at $125^{\circ} \mathrm{C}$ in the absence of an applied field. The effective perpendicular anisotropy field $\left(H_{\text {keff }}\right)$ measured from in-plane hysteresis loops reveals that the room-temperature $H_{\text {keff }}$ measurement (not shown) of $18 \mathrm{kOe}\left(1.4 \times 10^{6} \mathrm{~A} / \mathrm{m}\right)$ drops to about $8 \mathrm{kOe}(6.5$ $\times 10^{5} \mathrm{~A} / \mathrm{m}$ ) [as shown in Fig. $\left.1(\mathrm{c})\right]$ at $125{ }^{\circ} \mathrm{C}$. This value is still much higher than the in-plane demagnetization field at the same temperature $\left[4 \pi M_{s} \sim 650 \mathrm{Oe}\left(5.2 \times 10^{4} \mathrm{~A} / \mathrm{m}\right)\right]$. Therefore, we can conclude that at $125{ }^{\circ} \mathrm{C}$ and in the absence of an applied magnetic field (i.e., no Zeeman energy), the demagnetization energy exceeds the exchange energy and the anisotropy energy causing a magnetization relaxation by breaking into domains with perpendicular orientation. ${ }^{6}$

One way to reach such a temperature $\left(\sim 125^{\circ} \mathrm{C}\right)$ high enough to trigger spontaneous demagnetization is to pattern micron-sized wires and apply a number of current pulses with a well-defined pulse width and amplitude to attain temperature increase due to Joule heating. These nanosecond scale exposures to elevated temperatures allow us to control the magnetization relaxation process. For this purpose we have patterned our $\mathrm{CoNi} / \mathrm{Pd}$ magnetic multilayer film into $2 \mu \mathrm{m}$ wide, $10-\mu \mathrm{m}$-long microwires and applied $3.2 \mathrm{~V}$ amplitude 7.4-ns-wide pulses (with $\sim 30$ ps rise and fall times) corresponding to a current density of $4.8 \times 10^{7} \mathrm{~A} / \mathrm{cm}^{2}$. Finite-element calculations (using measured temperaturedependent thermal conductivity ${ }^{7}$ and electrical resistivity values of the full film) of the temperature distribution in our microwires with such current pulses indicated that the temperature within the microwire reaches $125^{\circ} \mathrm{C}$ in less than $100 \mathrm{ps}$ after the current pulse is turned on and the heating is uniform to a good approximation reaching a maximum of $10 \%$ drop close to the contact pads. In order to experimentally check the attainable temperature with such current pulses, we used MFM whereby we applied such pulses in the presence of a commercially available magnetic tip (MESP-HM from Veeco) to intentionally induce reversal of domains due to the stray fields from the tip. Since the
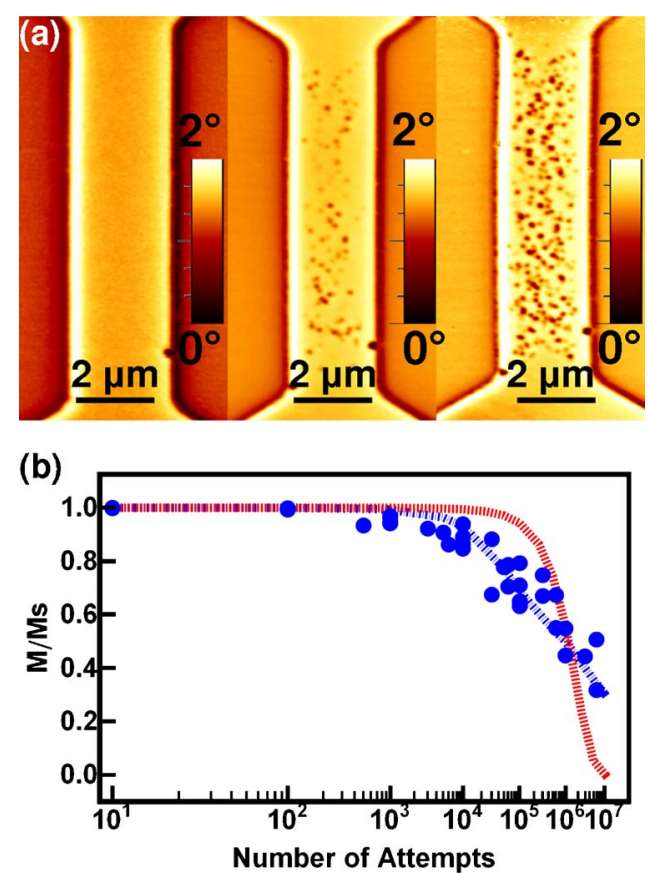

FIG. 2. (Color online) Mapping energy barrier distribution in CoNi-Pd multilayer microwires. (a) A series of demagnetization attempts using $7.4 \mathrm{ns,}$ $3.2 \mathrm{~V}$ pulses. From left to right: MFM image of the saturated state, MFM image after the application of a pulse train with 1000 pulses, and MFM image after the application of a pulse train with 10000 pulses. (b) Magnetization decay as a function of number of pulses applied (blue dots). Simple Arrhenius-Neel exponential decay fit (red dotted curve) and modified Arrhenius-Neel decay fit assuming 8\% Gaussian distribution of energy barriers (blue dot-dashed curve).

stray fields available from such tips are in the 500-700 Oe $\left(4 \times 10^{4}-5.6 \times 10^{4} \mathrm{~A} / \mathrm{m}\right)$ range depending on the exact tip geometry, ${ }^{8}$ it is expected that successful stray field-induced reversal requires the microwire coercivity to be reduced below 500 Oe $\left(4 \times 10^{4} \mathrm{~A} / \mathrm{m}\right)$ range which corresponds to $110-125{ }^{\circ} \mathrm{C}$ temperatures [in agreement with complementary finite-element method (FEM) calculations] according to the temperature dependent coercivity measurements presented in Fig. 1(b). In the following, the MFM tip is only used to image the magnetic configuration after the pulses have been turned off and is never engaged during the pulse application.

Figure 2(a) shows MFM images of the controlled demagnetization of the microwires by varying the number of pulses applied. As compared to the saturated state shown as the leftmost image on Fig. 2(a), one can see that increasing the number of pulses from $10^{4}$ (middle image) to $10^{5}$ (rightmost image) leads to more reversal events. In this experiment we have resaturated the wire magnetization, with a $2 \mathrm{~T}$ out-of-plane field in between each demagnetizing pulse train. Repeating such measurements for varying numbers of pulses spanning seven decades, we were able to go all the way from saturated state to almost fully demagnetized state. We also repeated the same measurements with different time intervals between consecutive pulses in the microsecond to millisecond range to check the consistency of thermal profile each time a pulse is applied and found that the results are independent of the pulse-to-pulse temporal intervals. This also agrees well with our FEM calculations suggesting that thermal time constant is expected to be in the $100 \mathrm{ps}$ range orders of magnitude below the pulse periods used in this experiment. 
All the MFM data collected were converted to gray scale and systematically threshold filtered, such that all pixel colors below a threshold value about roughly $75 \%$ in the gray scale were assigned a black color with all others being white. This procedure results in a black and white image where the black areas represent reversal domains. The magnetization decay can then be estimated from the ratio of the black areas to the wire area (i.e., $M / M_{s}=1-2\left(x / A_{\text {tot }}\right)$ where $M$ is the magnetization, $M_{s}$ is the saturation magnetization, $x$ is the total black area within the wire, and $A_{\text {tot }}$ is the total area of the wire). The level of magnetization decay extracted from the above analysis is shown in Fig. 2(b) where the blue dots are the experimental data plotted as a function of the number of pulses applied. Considering each such weak anisotropy region (i.e., nucleation volume) as a single domain, uniaxial particle Arrhenius-Neel formalism ${ }^{9}$ suggests that the magnetization relaxation obeys $M / M_{s}=\exp (-t / \tau)$ with $1 / \tau$ $=f_{0} \exp \left(-E_{B} / k_{B} T\right)$, where $t$ is the measurement time at a fixed temperature $T$.

In our case, $t=N t_{w}$ where $N$ is the number of pulses applied and $t_{w}$ is the pulse width of $7.4 \mathrm{~ns}$. The applied field is zero and the temperature is $125^{\circ} \mathrm{C}, \tau$ is the relaxation time, $f_{0}$ is the thermal attempt frequency $(\sim 1 \mathrm{GHz}), E_{B}$ $=K_{u} V_{\text {nucl }}\left(K_{u}\right.$ is the uniaxial anisotropy and $V_{\text {nucl }}$ is the nucleation volume) is the energy barrier for reversal and $k_{B}$ is the Boltzmann constant. The result of an Arrhenius-Neel fit to the data is shown as red dotted curve in Fig. 2(b). There is no agreement between the experimental data and the fit. However a very good agreement can be obtained by using a modified Arrhenius-Neel decay model assuming a Gaussian distribution of energy barriers (blue dot-dashed curve) with $8 \%$ sigma. From such a fit we are able to obtain $E_{B}$ $=0.55 \pm 0.05 \mathrm{eV}$ with $\langle\tau\rangle=12.5 \mathrm{~ms}$ in addition to the spatial map of the distribution of weak nucleation regions from individual MFM images. Furthermore, the energy barrier is given by $E_{B}=\frac{1}{2} H_{\text {keff }} M_{S} V_{\text {nucl }}$ (where nucleation volume $V_{\text {nucl }}$ $=l_{\text {nucl }}^{2}$. th with $t h$ being the magnetic film thickness) so that, using $M_{s}$ of $75 \mathrm{emu} / \mathrm{cm}^{3}(0.09 \mathrm{~T}), H_{\text {keff }}$ of $8 \mathrm{kOe}(6.4$ $\times 10^{5} \mathrm{~A} / \mathrm{m}$ ) at $125^{\circ} \mathrm{C}$ from (Fig. 1) and $31 \mathrm{~nm}$ magnetic film thickness, we can estimate a nucleation length $l_{\text {nucl }}$ of $10-15 \mathrm{~nm}$.

In order to check the validity of our method, we also followed the method of Thomson et $\mathrm{ll}^{4}{ }^{4}$ The SFD and reversal field measurements on $50 \mathrm{~nm}-10 \mu \mathrm{m}$ diameter prepatterned $\mathrm{CoNi} / \mathrm{Pd}$ multilayer islands when fit to the modified Sharrock formalism gave the best fit parameters of $l_{\text {nucl }}$ $=20 \mathrm{~nm}$ and $\sigma_{k}$ (anisotropy distribution assuming constant nucleation volume or equivalently energy barrier distribution) of $10.5 \%$. Only a small difference is obtained between the results of the two methods.

To investigate the variations in domain size during the cooling time, we can compare the domain areas obtained from different early stages of demagnetization attempts with 100 current pulses [Fig. 3(a)] and 500 current pulses [Fig. 3(b)]. Comparing similar areas in Figs. 3(a) and 3(b) (red circles) we see that the domain size variation after nucleation is minimal. The magnetization relaxation under these conditions is dominated by a domain nucleation process rather than propagation (especially because there is no external magnetic field). Given that the typical domain wall velocity in the absence of an applied field in such strongly exchange-

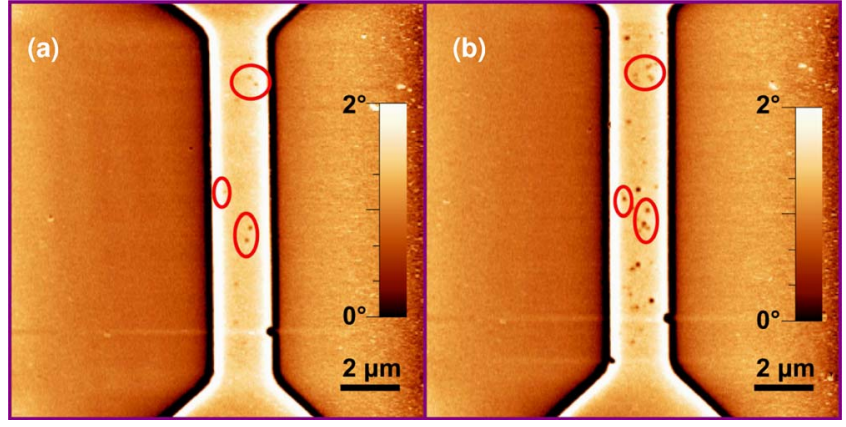

FIG. 3. (Color online) Nucleation and growth in the early stages of demagnetization. (a) MFM image after the application of 100 pulses with 7.4 ns width and $3.2 \mathrm{~V}$ amplitude. (b) MFM image after the application of 500 pulses with $7.4 \mathrm{~ns}$ width and $3.2 \mathrm{~V}$ amplitude.

coupled films is of the order of $10 \mathrm{~s}$ of meters per second, ${ }^{10}$ the domain wall motion during the cooldown is expected to be less than a nanometer. Therefore the magnetic configuration at the elevated temperature of $125^{\circ} \mathrm{C}$ is effectively quenched and the error introduced by the change in domain sizes during cooldown to room temperature is negligible. It is worthwhile to mention that since the microwires are initially uniformly magnetized, there is no spin torque effect that would lead to domain nucleation. Furthermore since the oersted field from the applied current points uniformly in plane along the wire and reaches a maximum value of $\sim 100$ Oe $\left(7.9 \times 10^{3} \mathrm{~A} / \mathrm{m}\right)$, while it may have a minor perturbative effect on the average energy barrier, its effect on the extracted energy barrier distribution is negligible.

In conclusion we have presented a measurement technique to determine the activation energy barrier distribution in strongly exchange-coupled magnetic multilayers. Even though this technique is strictly applicable to high temperature conditions, results from an independent method using bit patterned assembly confirm that it can as well provide a good approximation of the distribution at room temperature. This quantitative information on energy barrier distribution together with spatial mapping is applicable for future nanoscale memories development such as patterned media recording, MRAM or racetrack memory.

The authors would like to thank Elisabeth Dobisz for providing prepatterned substrates for the nanoisland reversal experiment and Tom Thomson for providing the code for Sharrock fits.

${ }^{1}$ J. M. Shaw, S. E. Russek, T. Thomson, M. J. Donahue, B. D. Terris, O. Hellwig, E. Dobisz, and M. L. Schneider, Phys. Rev. B 78, 024414 (2008)

${ }^{2}$ O. Ozatay, P. G. Gowtham, K. W. Tan, J. C. Read, K. A. Mkhoyan, M. G. Thomas, G. D. Fuchs, P. M. Braganca, E. M. Ryan, K. V. Thadani, J. Silcox, D. C. Ralph, and R. A. Buhrman, Nature Mater. 7, 567 (2008).

${ }^{3}$ P. A. Voltairas, D. I. Fotiadis, and C. V. Massalas, J. Magn. Magn. Mater. 217, L1 (2000)

${ }^{4}$ T. Thomson, G. Hu, and B. D. Terris, Phys. Rev. Lett. 96, 257204 (2006).

${ }^{5}$ M. P. Sharrock and J. T. McKinney, IEEE Trans. Magn. 17, 3020 (1981).

${ }^{6}$ O. Hellwig, A. Berger, J. B. Kortright, and E. E. Fullerton, J. Magn. Magn. Mater. 319, 13 (2007).

${ }^{7}$ Thermal conductivites were measured using the picosecond thermoreflectance technique by D. G. Cahill.

${ }^{8}$ M. Jaafar, A. Asenjo, and M. Vazquez, IEEE Trans. Nanotechnol. 7, 245 (2008).

${ }^{9}$ W. F. Brown, Jr., Phys. Rev. 130, 1677 (1963).

${ }^{10}$ A. Lyberatos and J. Ferre, J. Phys. D: Appl. Phys. 33, 1060 (2000). 